\title{
PERIODIC SOLUTIONS FOR NONAUTONOMOUS DIFFERENTIAL EQUATIONS AND INCLUSIONS IN TUBES
}

\author{
GRZEGORZ GABOR
}

Received 6 September 2002

We study the existence of periodic trajectories for nonautonomous differential equations and inclusions remaining in a prescribed compact subset of an extended phase space. These sets of constraints are nonconvex right-continuous tubes not satisfying the viability tangential condition on the whole boundary. We find sufficient conditions for existence of viable periodic trajectories studying properties of the exit subset of the tube. A new approximation approach for continuous multivalued maps is presented.

\section{Introduction}

The present paper is devoted to the existence of solutions to the boundary value problem

$$
\begin{gathered}
\dot{x}(t) \in F(t, x(t)), \quad \text { a.e. in }[0, T], \\
x(0)=x(T),
\end{gathered}
$$

or, in particular,

$$
\begin{gathered}
\dot{x}(t)=f(t, x(t)), \quad \text { a.e. in }[0, T], \\
x(0)=x(T),
\end{gathered}
$$

in a finite-dimensional space, with some additional state constraints, that is, we insist that trajectories do not leave a prescribed closed set $W \subset[0, T] \times \mathbb{R}^{n}$, or in other words, that trajectories are viable in $W$.

Following the literature in the subject, we call such trajectories viable periodic. If the right-hand side $F$ [resp., $f$ ] is $T$-periodic, then we can extend solutions of problem (1.1) [resp., (1.2)] to usual $T$-periodic trajectories. We concentrate in the paper on the problem defined for $t \in[0, T]$, leaving an unbounded $T$-periodic case as a standard consequence.

If $W=[0, T] \times \mathbb{R}^{n}$, there have been many papers dealing with the periodic problem in both single-valued and multivalued case. The methods have been mainly based on the degree theory (or fixed point index theory) applied to the single-valued or multivalued 
Poincaré operator of translation along trajectories. The guiding function method proposed by Krasnosel'skiu [10] has been successfully adopted for inclusions (1.1) (see, e.g., $[7,9]$ ) as well as for functional differential inclusions (see, e.g., [8] and the references therein). The main reason that we can use this method in a multivalued case is that the solution map very often has compact $R_{\delta}$ values, so that its composition with the evaluation map is sufficiently good to apply the degree theory.

When we add some essential state constraints, an important role in a successful solution of the viable $T$-periodic problem is played by studying the behavior of the right-hand side $F$ on the boundary $\partial W$ of $W$. To do this, we can use terms of tangent cones to the set $W$.

In the simplest case, if $F$ is sufficiently regular and satisfies the condition

$$
\{1\} \times F(t, x) \subset T_{W}(t, x) \quad \text { for every }(t, x) \in \partial W \backslash(\{T\} \times \operatorname{Int} W(T)),
$$

where $T_{W}(\cdot)$ stands for the Bouligand contingent cone and $W(t):=\left\{x \in \mathbb{R}^{n} \mid(t, x) \in\right.$ $W\}$, then no trajectory leaves $W$ and each $T$-periodic solution obtained by applying any standard technique is viable, so it is what we look for.

When, instead of (1.3), one has

$$
(\{1\} \times F(t, x)) \cap T_{W}(t, x) \neq \varnothing \quad \text { for every }(t, x) \in \partial W \backslash(\{T\} \times \operatorname{Int} W(T)),
$$

the above technique can be used if the operator $S_{W}: W(0) \multimap C\left([0, T], \mathbb{R}^{n}\right)$,

$$
S_{W}\left(x_{0}\right):=\left\{x \in C\left([0, T], \mathbb{R}^{n}\right) \mid x \text { is a solution to (1.1) viable in } W\right\}
$$

has sufficiently regular values. We note a pioneering paper on this matter for nonconvex tubes, namely [13], where the author has proved that for any Marchaud map and $W=$ $[0, T] \times K$ with $K$ being a proximal retract, the map $S_{W}$ is upper semicontinuous with compact $R_{\delta}$ values. The Scorza-Dragoni type arguments can be also applied (see [12]). As far as the author knows, the most general results in this direction have been recently obtained by Kryszewski and his coauthors (see [11] and the references therein) for sets defined via functional constraints.

The case where condition (1.4) is not satisfied everywhere becomes much worse because there is no hope to obtain sufficiently good topological properties of the map $S_{W}$. The so-called exit set $W^{-}$appears, where each trajectory leaves the set $W$ immediately. This set was first studied by Ważewski [16] in 1947 in the context of existence of viable trajectories in a given set. The Ważewski retract method has been intensively developed and used, for example, in the Conley index theory. It occurs that this exit set $W^{-}$can be also used to find viable $T$-periodic trajectories for nonautonomous differential equations.

The suitable topological method has been presented in [14]. The author has studied (1.2) with $f$ being $T$-periodic with respect to the first variable. The pair $\left(W, W^{-}\right)$has been assumed to be a $T$-periodic pair, which means that both $W$ and $W^{-}$are invariant with respect to some $T$-periodic process in $\mathbb{R}^{n}$ (see a definition of a process in Section 2 ). Since $\left(W, W^{-}\right)$is $T$-periodic, we can consider $W$ as a torus in $S^{1} \times \mathbb{R}^{n}$. Then, the techniques used in the Conley index theory and for autonomous differential equations may 
be applied. The main result in [14] has given the formulae joining the fixed point index of the Poincaré translation operator $\varphi_{(0, T)}(\cdot)$ and the number $\Lambda\left(W, W^{-}\right)$induced by the process generating the tube. The method proposed in [14] was used to obtain a lower bound of the number of periodic trajectories (see [17]) or chaotic dynamics (see $[15,18])$.

In the present paper, we develop the technique from [14] in some new directions. We avoid the assumption that $\left(W, W^{-}\right)$is a $T$-periodic pair. Since we do not assume that $f(\cdot, x)$ is $T$-periodic, we cannot use the identification of the tube with any torus. The main formulae (3.22) giving a connection between the index of $\varphi_{(0, T)}(\cdot)$ and the number $\Lambda\left(W, W^{-}\right)$defined in Proposition 3.10 is obtained without the assumption that $\left(W, W^{-}\right)$ is an isolating block (see [14]).

What is important is that our development also involves differential inclusions with continuous right-hand sides for which we obtain the existence results using the number $\Lambda\left(W, W^{-}\right)$.

We explain how the paper is organized.

In Section 2, we give some basic information which is used in the sequel. In particular, some properties of exit sets are presented.

In Section 3.1, we introduce the notion of a right-continuous tube and study its main properties together with relations between such tubes and their exit sets. In particular, we obtain a useful homotopy extension result, Proposition 3.9. We also define a number $\Lambda\left(W, W^{-}\right)$which is a main tool in existence results.

Main results on viable $T$-periodic trajectories for differential equations are presented in Section 3.2. Several corollaries and illustrations are also given.

In Section 4, we deal with differential inclusions of the form (1.1) with continuous right-hand sides. Under some regularity assumptions on the tube, we present the new method of studying an existence of viable $T$-periodic trajectories by a special approximation of the map $F$ by Lipschitz single-valued maps having the same exit set $W^{-}$.

\section{Preliminaries}

We start with some notations we use in the paper. By $\operatorname{Int} A, \operatorname{cl} A$, and $\partial A$ we mean, respectively, the interior, closure, and boundary of a subset $A$ of a metric space $X$. The open ball centered at $x_{0}$ and with radius $r$ is denoted by $B\left(x_{0}, r\right)$. For simplicity, the open unit ball in $\mathbb{R}^{n}$ is denoted by $B_{1}$. The distance between two sets $N, M \subset X$ is the number $\operatorname{dist}(N, M):=\inf \{d(x, y) \mid x \in N, y \in M\}$. If $N=\{x\}$, then we simply write $d_{M}(x):=\operatorname{dist}(\{x\}, M)$. We use the symbol $|\cdot|$ for the Euclidean norm. For any point $x$ of a metric space $X$, by $C(x)$ we denote the connected component containing $x$, that is, a maximal connected subset of $X$ which contains $x$.

Recall that a multivalued map $F: \mathbb{R} \times \mathbb{R}^{n} \multimap \mathbb{R}^{n}$ is a Marchaud map if $F$ has nonempty compact convex values, the map $F(\cdot, x)$ is measurable for every $x \in \mathbb{R}^{n}, F(t, \cdot)$ is upper semicontinuous for almost all $t \in \mathbb{R}$, and $F$ has at most the linear growth, which means that "there exists a locally integrable function $\beta: \mathbb{R} \rightarrow[0, \infty)$ such that $|F(t, x)|:=$ $\sup \{|y| ; y \in F(t, x)\} \leq \beta(t)(1+|x|)$ for every $(t, x) \in \mathbb{R} \times \mathbb{R}^{n}$."

It is known (see [6]) that, for any Marchaud map, the Cauchy problem (1.1) with $x\left(t_{0}\right)=x_{0}$ has a solution extensible onto the whole real line. By a solution to the inclusion 
(1.1) we always mean an absolutely continuous map. A solution will be often named a trajectory.

Let $W \subset \mathbb{R} \times \mathbb{R}^{n}$ be a closed set. We say that a trajectory $x(\cdot)$ starting from $\left(t_{0}, x_{0}\right)$ for the inclusion (1.1) is viable in $W$ if $(t, x(t)) \in W$ for every $t \geq t_{0}$ such that $W(t):=\{z \in$ $\left.\mathbb{R}^{n} \mid(t, z) \in W\right\} \neq \varnothing$.

For (1.2), assume that $f: \mathbb{R} \times \mathbb{R}^{n} \rightarrow \mathbb{R}^{n}$ is measurable with respect to the first variable and Lipschitz with respect to the second one in the sense that there is a locally integrable function $\alpha: \mathbb{R} \rightarrow[0, \infty)$ such that

$$
|f(t, x)-f(t, y)| \leq \alpha(t)|x-y|, \quad \text { for any } t \in \mathbb{R}, \quad x, y \in \mathbb{R}^{n} .
$$

Of course, if $f(\cdot, 0)$ is locally integrable, then the Cauchy problem for $(1.2)$ and any $\left(t_{0}, x_{0}\right) \in \mathbb{R} \times \mathbb{R}^{n}$ has a unique solution defined on the whole real line. Then, the global process on $\mathbb{R}^{n}$ is defined, that is, a continuous map $\varphi: \mathbb{R} \times \mathbb{R}^{n} \times \mathbb{R} \rightarrow \mathbb{R}^{n}$ with properties

(i) $\varphi_{(\sigma, 0)}(\cdot)=$ id for every $\sigma \in \mathbb{R}$;

(ii) $\varphi_{(\sigma, s+t)}=\varphi_{(\sigma+s, t)} \circ \varphi_{(\sigma, s)}$ for each $\sigma, s, t \in \mathbb{R}$.

It is seen that $\varphi_{(\sigma, t)}(x)$ is the value of the unique trajectory starting from $(\sigma, x)$ after a time $t$.

In what follows, we assume that $W \subset \mathbb{R}^{n}$ is a closed subset and $F: \mathbb{R}^{n} \multimap \mathbb{R}^{n}$ a Marchaud map.

On the boundary of $W$ we consider the following two sets.

Definition 2.1.

$$
\begin{aligned}
W_{s}(F):= & \left\{x_{0} \in \partial W \mid \text { each trajectory starting from } x_{0} \text { leaves } W \text { immediately }\right\}, \\
W_{e}(F):= & \left\{x_{0} \in \partial W \mid \text { there is a trajectory starting from } x_{0} \text { which leaves } W\right. \\
& \text { immediately }\} .
\end{aligned}
$$

A trajectory $x(\cdot)$ leaves $W$ immediately, if

$$
\exists t_{m}>0, \quad t_{m} \longrightarrow 0: x\left(t_{m}\right) \notin W .
$$

We will write briefly $W_{s}, W_{e}$ instead of $W_{s}(F), W_{e}(F)$. It is seen that for (1.2) generating a global process, $W_{s}=W_{e}$.

Since we use in this paper the exit set $W_{s}$, we recall some basic properties of it given in terms of tangent cones. By a tangent cone to a set $W$ in a point $x \in W$, we mean the Bouligand contingent cone defined as

$$
T_{W}(x):=\left\{v \in \mathbb{R}^{n} \mid \liminf _{h \rightarrow 0^{+}} \frac{\operatorname{dist}(x+h v, W)}{h}=0\right\} .
$$

Define

$$
W_{0}:=\left\{x \in \partial W \mid F(x) \cap T_{W}(x)=\varnothing\right\}
$$

We summarize properties of the exit set in the following. 
Proposition 2.2. (1) For any Marchaud map, $W_{0} \subset W_{s} \subset \mathrm{cl} W_{0}$.

(2) Let $F$ be a Marchaud map locally Lipschitz around $x \in \operatorname{cl} W_{0} \backslash W_{0}$. Denote $D_{W}(x):=$ $\mathbb{R}^{n} \backslash T_{\mathbb{R}^{n} \backslash W}(x)$.

If

$$
F(x) \cap\left(D_{W}(x) \cup T_{\partial W \backslash W_{0}}(x)\right)=\varnothing,
$$

then $x \in W_{s}$.

If

$$
F(x) \cap D_{W}(x) \neq \varnothing \quad \text { or } \quad F(x) \cap T_{W_{0}}(x)=\varnothing,
$$

then $x \notin W_{s}$.

This result was announced in [4]. For the complete proof, see [5]. It brings an important information from the practical point of view. It allows us to check whether the set $W_{s}$ is closed. As we will see, this is a standard assumption in problems which we investigate.

If (1.2) satisfies the uniqueness property, we can define the exit function $\tau: W \rightarrow[0, \infty]$,

$$
\tau(\sigma, x):=\sup \left\{t \geq 0 \mid\left(\sigma+t, \varphi_{(\sigma, t)}(x)\right) \in W\right\} .
$$

It is easy to check that, if $W_{s}$ is closed, the exit function is continuous.

Throughout the paper, we will use well-known notions of absolute (and absolute neighborhood) retracts denoted by AR and ANR, respectively. For properties of ARs and ANRs, see, for example, [2]. Obviously, if a closed set $X \subset \mathbb{R}^{n}$ is an AR (resp., ANR), then it is a retract of $\mathbb{R}^{n}$ (resp., of its open neighborhood in $\mathbb{R}^{n}$ ).

If $X$ is a compact ANR, then, for any continuous map $f: X \rightarrow X$, the Lefschetz number $\lambda(f)$ of $f$ is well defined (see [9] for details). Note that $\lambda\left(\mathrm{id}_{X}\right)=\chi(X)$, the Euler characteristic of $X$.

For compact ANRs and continuous maps, the fixed point index is also constructed (see [3]) and has the following properties.

Let $U, V, U_{1}, U_{2}$ be open subsets of $X$ and $f, g: X \rightarrow X$. Denote by $\operatorname{Fix}(f)$ the set of fixed points of $f$.

Existence 2.3. If $\operatorname{Fix}(f) \cap \partial U=\varnothing$ and ind $(f, U) \neq 0$, then there is a fixed point of $f$ in $U$. Localization (Excision) 2.4. If $\operatorname{Fix}(f) \subset V \subset U$, then $\operatorname{ind}(f, U)=\operatorname{ind}(f, V)$.

Additivity 2.5. If $U_{1} \cap U_{2}=\varnothing$ and $\operatorname{Fix}(f) \cap\left(\operatorname{cl} U \backslash\left(U_{1} \cup U_{2}\right)\right)=\varnothing$, then $\operatorname{ind}(f, U)=$ $\operatorname{ind}\left(f, U_{1}\right)+\operatorname{ind}\left(f, U_{2}\right)$.

Homotopy 2.6. If $h: X \times[0,1] \rightarrow X$ is such that $h(\cdot, 0)=g, h(\cdot, 1)=f$ and $\operatorname{Fix}(h(\cdot, t)) \cap$ $\partial U=\varnothing$ for every $t \in[0,1]$, then ind $(f, U)=\operatorname{ind}(g, U)$.

Normalization 2.7. ind $(f, X)=\lambda(f)$.

From the localization property it follows that, for any isolated subset $P$ of $\operatorname{Fix}(f)$, we can define ind $(f, P)$ as the index on some sufficiently small open neighborhood of $P$ in $X$. 
In this paper, we will use the following notion.

Definition 2.8. Let $(X, Y)$ be a topological pair (it means that $X$ is a topological space and $Y$ its closed subset) and let $(A, B)$ be a subpair, that is, a pair such that $A \subset X$ and $B \subset Y$. The pair $(A, B)$ is a strong deformation retract pair of $(X, Y)$, if there is a map of topological pairs (the map $f:(A, B) \rightarrow(C, D)$ is a map of pairs, if $f(A) \subset C$ and $f(B) \subset$ $D), h:(X \times[0,1], Y \times[0,1]) \rightarrow(X, Y)$ such that

(i) $h(x, 0)=x$ for every $x \in X$;

(ii) $h(x, 1) \in A$ for every $x \in X$, and $h(x, 1) \in B$ for every $x \in Y$;

(iii) $h(x, s)=x$ for every $x \in A$ and $s \in[0,1]$.

If $Y=B=\varnothing$, then $A$ is a strong deformation retract of $X$.

\section{Differential equations on right-continuous tubes}

In this section, we deal with differential equations (1.2) with measurable Lipschitz righthand side $f$. The aim is to find a $T$-periodic trajectory for (1.2), that is, with $x(0)=x(T)$, satisfying simultaneously state constraints, that is, remaining in a prescribed set $W \subset$ $[0, T] \times \mathbb{R}^{n}$.

In the first part of this section, we show what sets of constraints will be dealt with. We give several properties describing the connection between dynamics and topological properties of some subsets of $W$.

In the second subsection, we present the main result on the existence of a viable periodic trajectory for (1.2) and some corollaries (see Corollaries 3.12-3.19). Some illustrating examples are given.

3.1. Right-continuous tubes. Let $J$ be an arbitrary interval in $\mathbb{R}$, bounded or not. We start with the following notion.

Definition 3.1. The closed set $W \subset J \times \mathbb{R}^{n}$ is a right-lower semicontinuous tube, if the map $J \ni t \mapsto W(t):=\left\{x \in \mathbb{R}^{n} \mid(t, x) \in W\right\}$ has nonempty closed values and satisfies the following condition:

(i) for every $t_{0} \in J, x_{0} \in W\left(t_{0}\right)$ and each sequence $\left(t_{n}\right) \subset\left[t_{0}, \infty\right)$ converging to $t_{0}$, there exists a sequence $\left(x_{n}\right)$ such that $x_{n} \in W\left(t_{n}\right)$ and $x_{n} \rightarrow x_{0}$.

If the map $W(\cdot)$ is also upper semicontinuous, then we call this tube right-continuous. We say that the tube $W$ is $T$-periodic, if the length of $J$ is not less that $T$ and $W(t)=W(t+T)$ for each $t, t+T \in J$.

It is seen that each compact right-lower semicontinuous tube and each continuous tube is right-continuous.

It is also obvious that if we assume that

$f$ is $T$-periodic with respect to the first variable,

then each $T$-periodic tube $W \subset[0, T] \times \mathbb{R}^{n}$ can be considered a subset of a $T$-periodic tube defined on the whole real line. 
For (1.2) and any continuous tube $W \subset[0, T] \times \mathbb{R}^{n}$, notice that $\{T\} \times W(T)$ is a subset of the exit set $W_{s}$ of $W$ (see Definition 2.1) for the map $(t, x) \mapsto(1, f(t, x))$. We define

$$
\begin{aligned}
W^{-} & :=\left(W_{s} \backslash(\{T\} \times W(T))\right) \\
& \cup\left\{(T, x) \in\{T\} \times W(T) \mid \exists\left(t_{m}, x_{m}\right) \in W_{s} \backslash(\{T\} \times W(T)):\left(t_{m}, x_{m}\right) \longrightarrow(T, x)\right\} .
\end{aligned}
$$

If $f$ satisfies (3.1), then, for any $T$-periodic tube $W \subset[0, T] \times \mathbb{R}^{n}$ with $W^{-}(0)=$ $W^{-}(T)$, the set $W^{-}$is the intersection of $W$ and the exit set for the tube extended on $[0, \infty)$.

Throughout our considerations, we will assume that

$W$ is a compact right-continuous tube and $W^{-}$is closed.

It is seen that if condition (3.3) holds, then $W^{-}=\operatorname{cl}\left(W_{s} \backslash(\{T\} \times W(T))\right)$.

Lemma 3.2. If condition (3.3) is satisfied and $W^{-} \neq \varnothing$, then $W^{-}(T) \neq \varnothing$.

Proof. Suppose that $W^{-} \neq \varnothing$ and $W^{-}(T)=\varnothing$. The set $A:=\left\{t \in[0, T] \mid W^{-}(t) \neq \varnothing\right\}$ is closed, since $W^{-}$is compact. Denote $t_{e}=\sup A$ and take $x_{e} \in \mathbb{R}^{n}$ such that $\left(t_{e}, x_{e}\right) \in A$.

Since $W(\cdot)$ is right-continuous, there is a sequence $\left(t_{m}, x_{m}\right) \in W$ such that $t_{e}<t_{m}<T$ and $\left(t_{m}, x_{m}\right) \rightarrow\left(t_{e}, x_{e}\right)$. Therefore, for any $m \geq 1$, the trajectory $\varphi_{\left(t_{m}, \cdot\right)}\left(x_{m}\right)$ remains in $W$ up to $T$. Since $\left(t_{e}, x_{e}\right) \in W^{-}$, this is impossible due to Filippov's theorem.

If the set $W^{-}$is more regular, we can obtain better information on its shape. To this end, for each $(\sigma, x) \in W^{-}$, take the connected component $C(\sigma, x)$ in $W^{-}$containing $(\sigma, x)$ and define

$$
\theta(\sigma, x):=\sup \left\{t \in[0, T] \mid C(\sigma, x) \cap W^{-}(t) \neq \varnothing\right\} .
$$

Lemma 3.3. Under assumption (3.3), if $W^{-}$has only a finite number of connected components, then $\theta(\sigma, x)=T$ for every $(\sigma, x) \in W^{-}$.

Proof. Suppose, on the contrary, that for some $(\sigma, x) \in W^{-}, \theta:=\theta(\sigma, x)<T$. Since $C(\sigma, x)$ is compact, there is $z \in \mathbb{R}^{n}$ such that $(\theta, z) \in C(\sigma, x)$ and $d_{\left(W^{-} \backslash C(\sigma, x)\right)}(\theta, z)>0$. Therefore, there are $\varepsilon, \delta>0$ such that, for every $(\beta, u) \in W$ with $\beta>\theta$ and $|(\theta, z)-(\beta, u)|<\delta$, we have $\tau(\beta, u) \geq \varepsilon$. But this is impossible, since $\tau$ is continuous and $\tau(\theta, z)=0$.

Define

$$
P_{W}:=\left\{x \in W(0) \mid \varphi_{(0, T)}(x)=x, \varphi_{(0, t)}(x) \in W(t) \text { for every } t \in[0, T]\right\} .
$$

In many places we will assume that

$$
P_{W} \cap \partial(W(0) \cap W(T))=\varnothing .
$$

We can prove the following important property.

Lemma 3.4. Under assumptions (3.3) and (3.6) the set $P_{W}$ is isolated in the fixed point set of $\varphi_{(0, T)}(\cdot)$. 
Proof. The set $P_{W}$ is obviously closed, since $W$ is closed. It is sufficient to prove that $\{t\} \times \varphi_{(0, t)}\left(P_{W}\right) \subset \operatorname{Int} W$ for every $t \in[0, T]$.

Let $x \in P_{W}$, Then, $x \in \operatorname{Int}(W(0) \cap W(T))$. For every $m \geq 1$, we can choose an open neighborhood $V_{m} \subset \operatorname{Int} W(0)$ of $x$ such that

$$
\left|\varphi_{(0, t)}(z)-\varphi_{(0, t)}(x)\right| \leq \frac{1}{m} \quad \text { for any } z \in V_{m}, t \in[0, T]
$$

Suppose, on the contrary, that there is $0<t_{0}<T$ such that $\left\{t_{0}\right\} \times \varphi_{\left(0, t_{0}\right)}(x) \in \partial W$. Since $\varphi(0, t)$ is a homeomorphism, we can see that the set

$$
\Omega:=\left\{\left(s, \varphi_{(0, s)}(z)\right) \mid z \in V, t_{0}-\delta<s<t_{0}+\delta<T\right\}
$$

is an open neighborhood of $\left\{t_{0}\right\} \times \varphi_{\left(0, t_{0}\right)}(x)$ in $\mathbb{R} \times \mathbb{R}^{n}$. Therefore, there are $\left(t_{m}, y_{m}\right) \in$ $\Omega \backslash W$, which implies that there are $x_{m} \in V$ with $y_{m}=\varphi_{\left(0, t_{m}\right)}\left(x_{m}\right)$, and the trajectory starting from $\left(0, x_{m}\right)$ leaves $W$ at some point $\left(\tau\left(0, x_{m}\right), \varphi_{\left(0, \tau\left(0, x_{m}\right)\right.}\left(x_{m}\right)\right)$ near $\left(t_{0}, \varphi_{\left(0, t_{0}\right)}(x)\right)$. Taking $m \rightarrow \infty$, we conclude that $\left(t_{0}, \varphi_{\left(0, t_{0}\right)}(x)\right) \in W^{-}$; a contradiction.

There may be some points in $\partial W$ with $p=(t, x) \in\{t\} \times \operatorname{Int} W(t)$, while each point from $\{t\} \times \partial W(t)$ belongs to $\partial W$. Therefore, the lemma above leads us to the following necessary condition for the existence of viable periodic trajectories.

Corollary 3.5. Let (3.3) and (3.6) be satisfied. If there is a viable T-periodic trajectory in $W$, then Int $W(t) \neq \varnothing$ for every $t \in[0, T]$.

The natural question arises: is it possible to verify condition (3.6) without following along all trajectories starting from $\partial(W(0) \cap W(T))$ on the interval $[0, T]$ ? It occurs that we can find some sufficient conditions in terms of tangent cones, as we can see below.

At first, define

$$
W^{0}:=\left\{(\sigma, x) \in \partial W \backslash W^{-} \mid(1, f(\sigma, x)) \in T_{\partial W}(\sigma, x)\right\}
$$

Proposition 3.6. If (3.3) is satisfied,

$$
W(T) \subset W(0), \quad W^{-}(T) \subset W^{-}(0),
$$

and there is no pathwise connected component $D$ of $W^{0}$ joining $D(0)$ and $D(T)$, then condition (3.6) holds true. (Recall that a nonempty subset $A$ of a topological space $X$ is a pathwise connected component of $X$, if $A$ is a maximal subset such that each two points $x, y \in A$ may be joined by a continuous path $\alpha:[0,1] \rightarrow A$, that is, $\alpha(0)=x$ and $\alpha(1)=y$.)

Proof. Suppose that $x \in P_{W} \cap \partial(W(0) \cap W(T))$. We can prove that $\left(t, \varphi_{(0, t)}(x)\right) \in \partial W$ for every $t \in[0, T]$. Indeed, if not, then as in the proof of Lemma 3.4, one shows that there is a time $t_{0} \in[0, T]$ at which the trajectory meets $W^{-}$. The only possibility is that $t_{0}=T$, but then $x \in W^{-}(T) \subset W^{-}(0)$ in contrary to the fact that from $x$ a viable trajectory starts.

Now, $\alpha:[0,1] \rightarrow W^{0}, \alpha(\lambda):=\varphi_{(0, \lambda T)}(x)$ is a path joining $(0, x)$ and $(T, x)$ in $W^{0}$; a contradiction. 


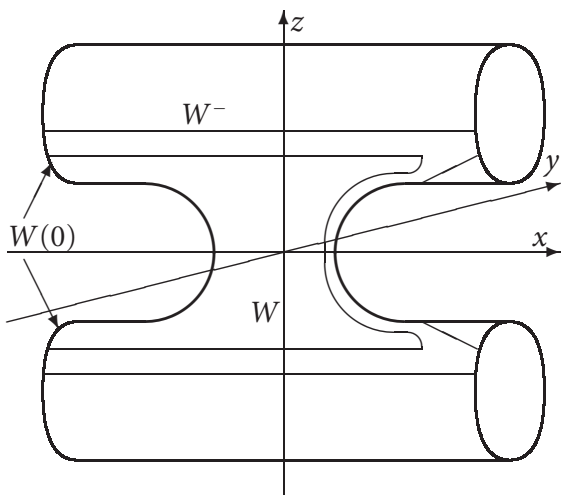

Figure 3.1

Corollary 3.7. If (3.3) and (3.10) are satisfied, and $W^{0}$ is totally disconnected, then condition (3.6) is satisfied. (The set is totally disconnected, if each connected component is a singleton.)

The following two results give the relation between properties of $W^{-}$and the shape of the whole tube $W$.

Proposition 3.8. Let assumption (3.3) be satisfied. Let $W^{-}$have a finite number of connected components, and let $\{T\} \times W^{-}(T)$ be a retract of $W^{-}$. If $W(T)$ is disconnected, then the whole tube $W$ is disconnected.

Proof. Let $W(T)=A_{1} \cup A_{2}$, where $A_{i}$ are disjoint, nonempty, and closed. Consider the connected components $B_{i}$ in $W$ containing $A_{i}$, respectively. Let

$$
\theta:=\inf \left\{t \geq 0 \mid B_{1}(t) \cap B_{2}(t)=\varnothing\right\}
$$

and suppose that $\theta>0$. We can easily prove that each point $(\theta, x) \in\{\theta\} \times\left(B_{1}(\theta) \cap B_{2}(\theta)\right)$ belongs to $W^{-}$. Indeed, if not, the trajectory starting from $(\theta, x)$ goes in one of the components, say $B_{1}$, during some positive time $t$. At the time $\theta+t$, we have $d:=\operatorname{dist}\left(B_{1}(\theta+\right.$ $\left.t), B_{2}(\theta+t)\right)>0$. Then, there is an open neighborhood $V$ of $(\theta, x)$ such that, for any $(\sigma, z) \in V \cap \partial W,\left|\varphi_{(\sigma, \theta+t-\sigma)}(z)-\varphi_{(\theta, t)}(x)\right|<d$. On the other hand, trajectories starting from points $(\sigma, z) \in V \cap B_{2}$ with $\sigma>\theta$ go inside $B_{2}$; a contradiction. From the proof of Lemma 3.3, it follows that $(\theta, x)$ may be joined by paths with both $A_{1}$ and $A_{2}$, which contradicts the assumption that $\{T\} \times W^{-}(T)$ is a retract of $W^{-}$. The proof is complete.

One can find an example (in $[0, T] \times \mathbb{R}^{2}$ ) that the result above is not true without the retracting assumption. The sets $W$ and $W^{-}$may look like in Figure 3.1.

The second proposition, a homotopy extension result, is crucial for our further considerations. 
1066

Periodic solutions in tubes

Proposition 3.9. If assumption (3.3) is satisfied and

$$
\{T\} \times W^{-}(T) \text { is a strong deformation retract of } W^{-},
$$

then this homotopy can be extended to a strongly deformation homotopy

$$
h:\left(W \times[0,1], W^{-} \times[0,1]\right) \longrightarrow\left(\{T\} \times W(T),\{T\} \times W^{-}(T)\right) .
$$

Moreover, for each trajectory viable in $W$,

$$
h\left(\left(t, \varphi_{(0, t)}(x)\right), \lambda\right)=\left(t+\lambda(T-t), \varphi_{(0, t+\lambda(T-t))}(x)\right) .
$$

In particular, $h((0, x), 1)=(T, x)$ for every $x \in P_{W}$.

Proof. Let $k: W^{-} \times[0,1] \rightarrow W^{-}$be a strong deformation homotopy onto $\{T\} \times W^{-}(T)$. Define $h: W \times[0,1] \rightarrow W$,

$$
\begin{aligned}
& h((\sigma, x), \lambda)= \\
& \qquad \begin{cases}\left(\sigma+\lambda(T-\sigma), \varphi_{(\sigma, \lambda(T-\sigma))}(x)\right), & \text { if }\left(\sigma+t, \varphi_{(\sigma, t)}(x)\right) \notin W^{-} \\
k\left(\left(\sigma+\tau(\sigma, x), \varphi_{(\sigma, \tau(\sigma, x))}(x)\right), \frac{\lambda(T-\sigma)-\tau(\sigma, x)}{T-\sigma-\tau(\sigma, x)}\right), & \text { for every } 0 \leq t \leq \lambda(T-\sigma),\end{cases}
\end{aligned}
$$

Notice that $h$ is continuous, $h(\cdot, 0)=\mathrm{id}_{W}$, and

$$
\begin{aligned}
& h((\sigma, x), 1)= \\
& \begin{cases}\left(T, \varphi_{(\sigma, T-\sigma)}(x)\right) \in\{T\} \times W(T), & \text { if }\left(\sigma+t, \varphi_{(\sigma, t)}(x)\right) \notin W^{-} \\
k\left(\left(\sigma+\tau(\sigma, x), \varphi_{(\sigma, \tau(\sigma, x))}(x)\right), 1\right) \in\{T\} \times W^{-}(T), & \text { for every } 0 \leq t \leq T-\sigma,\end{cases}
\end{aligned}
$$

Moreover, $h$ is an extension of $k$.

Denote

$$
K:=W(0), \quad K^{-}:=W^{-}(0)
$$

Then, the map $h(\cdot, 1)$ described above induces the following two maps:

$$
\begin{gathered}
H_{1}: K \longrightarrow K \quad \text { such that }\left(T, H_{1}(x)\right)=h((0, x), 1), \\
H_{1}^{-}: K^{-} \longrightarrow K^{-} \quad \text { such that }\left(T, H_{1}(x)\right)=h((0, x), 1) .
\end{gathered}
$$

Proposition 3.10. Assume that conditions (3.3), (3.10), and (3.12) are satisfied and

$$
W(0), W^{-}(0) \text { are ANRs. }
$$


Then, the number

$$
\Lambda\left(W, W^{-}\right):=\lambda\left(H_{1}\right)-\lambda\left(H_{1}^{-}\right)
$$

does not depend on the choice of a homotopy deforming $W^{-}$onto $\{T\} \times W^{-}(T)$.

Proof. At first, We note that the Lefschetz numbers $\lambda\left(H_{1}\right)$ and $\lambda\left(H_{1}^{-}\right)$are well defined because the sets $K$ and $K^{-}$defined in (3.17) are compact ANRs.

Let $k, \bar{k}$ be two homotopies strongly deforming $W^{-}$onto $\{T\} \times W^{-}(T)$ and $h, \bar{h}: W \times$ $[0,1] \rightarrow\{T\} \times W(T)$ their extensions. For each $x \in K$ and $s \in[0,1]$, we define $\chi(x, s) \in K$ as a state such that

$$
(T, \chi(x, s))=\bar{h}(h((0, x), s), 1)
$$

Notice that $(T, \chi(x, 0))=h((0, x), 1)=\left(T, H_{1}(x)\right)$ and $(T, \chi(x, 1))=\bar{h}((0, x), 1)=(T$, $\left.\bar{H}_{1}^{-}(x)\right)$. Moreover, $\chi$ is continuous. Thus, $\lambda\left(H_{1}\right)=\lambda\left(\bar{H}_{1}\right)$ and $\lambda\left(H_{1}^{-}\right)=\lambda\left(\bar{H}_{1}^{-}\right)$, and the proof is complete.

3.2. Periodic trajectories of differential equations. In this subsection, we present the main result on existence of viable periodic trajectories in continuous tubes and some of its consequences.

Theorem 3.11. Let assumptions (3.3), (3.10), (3.12), and (3.19) be satisfied. Then, $\Lambda$ ( $W$, $\left.W^{-}\right) \neq 0$ implies that there exists a viable $T$-periodic trajectory in $W$. Moreover, if condition (3.6) holds, then

$$
\text { ind }\left(\varphi_{(0, T)}, P_{W}\right)=\Lambda\left(W, W^{-}\right) \text {. }
$$

Proof. Using Proposition 3.9, we find a homotopy $h$ strongly deforming the pair $\left(W, W^{-}\right)$ onto $\left(\{T\} \times W(T),\{T\} \times W^{-}(T)\right)$. Consider a topological direct sum of $K$ and $K^{-} \times S^{1}$ ( $K$ and $K^{-}$are defined in (3.17)) and the quotient space $C$ made of this sum by identifying points $x \in K^{-}$with $(x, 1) \in K^{-} \times S^{1}$. Since $K$ and $K^{-}$are ANRs, so is $C$.

Denote, for any $p \in W$, by $\tilde{h}(p)$ the point in $W(T)$ such that $h(p, 1)=(T, \tilde{h}(p))$. Define $\chi C \times[0,1] \rightarrow C$ as follows: for $x \in K$,

$$
\chi(x, s):= \begin{cases}\tilde{h}\left((1-s) T, \varphi_{(0,(1-s) T)}(x)\right), & \text { if } \tau(0, x) \geq(1-s) T \\ \left(\tilde{h}\left(\tau(0, x), \varphi_{(0, \tau(0, x))}(x)\right), \exp \left(\left(1-s-\frac{\tau(0, x)}{T}\right) \pi i\right)\right), & \text { if not, }\end{cases}
$$

and, for $x=(z, u) \in K^{-} \times S^{1}$,

$$
\chi((z, u), s):=\left(H_{1}^{-}(z), u \exp ((1-s) \pi i)\right)
$$


Notice that, since $h\left(W^{-} \times\{1\}\right) \subset\{T\} \times K^{-}$, the only fixed points of $\chi_{0}:=\chi(\cdot, 0)$ could lie in $P_{W}$ or in $K^{-}$. But, for $x \in K^{-}$, we have

$$
\chi((z, u), 0)=\left(H_{1}^{-}(z),-u\right) \neq(z, u)
$$

which eliminates the latter case.

Consider the tube $\widehat{W} \subset[0, \infty) \times \mathbb{R}^{n}$ defined by

$$
\widehat{W}(t)= \begin{cases}W(t), & \text { if } t \in[0, T], \\ W(T), & \text { if } t>T,\end{cases}
$$

and $\hat{f}:[0, \infty) \times \mathbb{R}^{n} \rightarrow \mathbb{R}^{n}$,

$$
\hat{f}(t, x)= \begin{cases}f(t, x), & \text { if } t \in[0, T] \\ f(T, x), & \text { if } t>T .\end{cases}
$$

For this extended tube, we have $\widehat{W}_{s}=W^{-} \cup\left([T, \infty) \times W^{-}(T)\right)$. By $\tau$ we will mean the exit function for the tube $\widehat{W}$.

Consider the open neighborhood of $P_{W}$ in $K$ :

$$
V:=\{x \in W(0) \mid \tau(0, x)>T\}
$$

It is obvious that $\partial_{K} V \subset\{x \in W(0) \mid \tau(0, x)=T\}$. It implies, thanks to (3.10), that there are no fixed points of $\varphi_{(0, T)}$ on $\partial_{K} V$ and, if we assume (3.6), then also on $\partial V \subset \operatorname{Int} K$ (see Lemma 3.4). Moreover, the only fixed points of $\varphi_{(0, T)}$ in $V$ are points from $P_{W}$.

Note that $\left.\chi_{0}\right|_{V}=\left.\varphi_{(0, T)}\right|_{V}$, and hence, using Localization 2.4 and Normalization 2.7 properties of the fixed point index on ANRs, we obtain

$$
\lambda\left(\chi_{0}\right)=\operatorname{ind}\left(\left.\chi_{0}\right|_{V}, P_{W}\right)
$$

From the existence property of the index Existence 2.3, now it is sufficient to prove that $\lambda\left(\chi_{0}\right) \neq 0$. But $\lambda\left(\chi_{0}\right)=\lambda\left(\chi_{1}\right)$, where $\chi_{1}=\chi(\cdot, 1)$, since $\chi(\cdot, s)$ has no fixed points on $\partial_{K} V$ for every $s \in[0,1]$.

We compute $\lambda\left(\chi_{1}\right)$. Notice that

$$
\begin{gathered}
\chi_{1}(x)=H_{1}(x) \quad \text { for } x \in K, \\
\chi_{1}(z, u)=\left(H_{1}^{-}(z), u\right) \quad \text { for } x \in K^{-}, u \in S^{1} .
\end{gathered}
$$

Therefore, $\chi_{1}$ induces the following maps:

$$
\begin{array}{cc}
F_{1}: K \longrightarrow K, & F_{1}(x):=H_{1}(x), \\
F_{2}: K^{-} \times S^{1} \longrightarrow K^{-} \times S^{1}, & F_{2}(z, u):=\left(H_{1}^{-}(z), u\right), \\
F_{3}: K^{-} \longrightarrow K^{-}, & F_{3}(x):=H_{1}^{-}(x) .
\end{array}
$$


It is seen that $\lambda\left(F_{2}\right)=0$. Using the Mayer-Vietoris exact sequence of the triad $\left(C, K, K^{-} \times\right.$ $\left.S^{1}\right)$ and the exact sequence of the pair $\left(K, K^{-}\right)$we obtain

$$
\lambda\left(\chi_{1}\right)=\lambda\left(F_{1}\right)+\lambda\left(F_{2}\right)-\lambda\left(F_{3}\right)=\lambda\left(F_{1}\right)-\lambda\left(F_{3}\right) .
$$

The proof of the first statement is finished. To prove the second one it is sufficient to notice that under assumption (3.6), since there are no fixed points of $\varphi_{(0, T)}$ on $\partial V$, we have

$$
\text { ind }\left(\varphi_{(0, T)}, P_{W}\right)=\operatorname{ind}\left(\left.\varphi_{(0, T)}\right|_{V}, P_{W}\right)=\operatorname{ind}\left(\left.\chi_{0}\right|_{V}, P_{W}\right) \text {. }
$$

Thus, the proof of the theorem is complete.

Corollary 3.12. Let assumptions (3.3), (3.12), and (3.19) be satisfied for $W$ and $W^{-}$being $T$-periodic and $f$ being T-periodic with respect to the first variable. Then, $\Lambda\left(W, W^{-}\right) \neq 0$ implies that there exists a viable T-periodic trajectory in $W$. Moreover, if condition (3.6) is satisfied, then (3.22) holds.

Remark 3.13. Theorem 3.11 and the above result are generalizations of Theorem 7.1 in [14]. With comparison to [14], we do not assume that $\left(W, W^{-}\right)$is an isolating block, that is, the maximal invariant subset in $W$ is included in Int $W$, due to Lemma 3.4. The assumption in [14] that $\left(W, W^{-}\right)$is a $T$-periodic block, that is, there is a continuous process $\omega$ on $\mathbb{R}^{n}$ such that $W$ and $W^{-}$are invariant under the flow $\omega_{t}^{*}(\sigma, x):=\left(\sigma+t, \omega_{(\sigma, t)}(x)\right)$, is strictly related to our assumption (3.12). Indeed, for any $T$-periodic block we have the following strong deformation homotopy of $\left(W, W^{-}\right)$onto $\left(\{T\} \times W(T),\{T\} \times W^{-}(T)\right)$ :

$$
h((\sigma, x), s)=\omega_{(\sigma, s(T-\sigma))}(x)
$$

In Proposition 3.9, we explain the connection between deformation $W^{-}$onto $\{T\} \times$ $W^{-}(T)$ and $\left(W, W^{-}\right)$onto $\left(\{T\} \times W(T),\{T\} \times W^{-}(T)\right)$. The extended homotopy obtained in Proposition 3.9 obviously may not generate a global process but only a semiprocess (for positive times) and only on the tube, but this suffices. In particular, $W^{-}(0)$ may have more connected components than $W^{-}(T)$. The semiprocess on the extended tube may be given by

$$
\omega_{(\sigma, t)}(x)= \begin{cases}h\left((\sigma, x), \frac{t}{T-\sigma}\right), & \text { if } t \leq T-\sigma, \\ h\left(h_{1}^{k}(\sigma, x), \frac{t+\sigma-k T}{T}\right), & \text { if } t \in[k T-\sigma,(k+1) T-\sigma],\end{cases}
$$

where $h_{1}(\cdot):=h(\cdot, 1)$ and $h_{1}^{k}:=h_{1} \circ \cdots \circ h_{1}, k$ times.

Example 3.14. Consider the set

$$
W:=\left\{(t, x, y) \in \mathbb{R}^{3} \mid 0 \leq t \leq 6,0 \leq x \leq 2,0 \leq y \leq-\frac{1}{2} t+4\right\}
$$




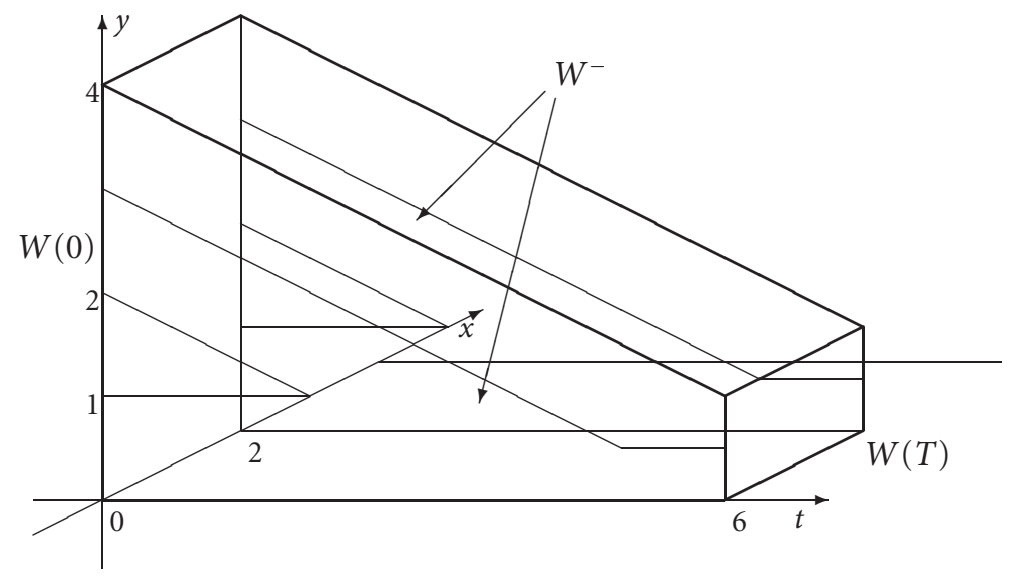

Figure 3.2.

and a right-hand side $f=\left(f_{1}, f_{2}\right)$ described on $\partial W$ by the inequalities

$$
\begin{gathered}
f_{2}(t, x, 0) \geq 0, \quad f_{2}\left(t, x,-\frac{1}{2} t+4\right) \leq-\frac{1}{2}, \\
f_{2}\left(t, i,-\frac{1}{2} t+3\right)<-\frac{1}{2} \quad \text { for } t \in[0,5), \quad f_{2}\left(t, i, \frac{1}{2}\right)<0 \quad \text { for } t \in[5,6], i \in\{0,2\}, \\
f_{2}\left(t, i,-\frac{1}{2} t+2\right)>-\frac{1}{2}, \quad f_{2}(t, i, 1)<0 \quad \text { for } t \in[0,2), i \in\{0,2\}, \\
f_{1}(t, 0, y)<0, \quad f_{1}(t, 2, y)>0 \quad \text { for } t \in[0,4), y \in[0,1), \\
f_{1}(t, 0, y)<0, \quad f_{1}(t, 2, y)>0 \quad \text { for } t \in[0,5], y \geq 0, y \in\left(-\frac{1}{2} t+2,-\frac{1}{2} t+3\right), \\
t \in[5,6], \quad y \in\left[0, \frac{1}{2}\right), \\
f_{1}(t, 0, y) \geq 0, \quad f_{1}(t, 2, y) \leq 0 \quad \text { for } t \in[0,2], y \in\left[1,-\frac{1}{2} t+2\right], \\
f_{1}(t, 0, y) \geq 0, \quad f_{1}(t, 0, y) \leq 0 \quad \text { for } t \in[0,5], y \in\left[-\frac{1}{2} t+3,-\frac{1}{2} t+4\right], \\
t \in[5,6], \quad y \in\left[\frac{1}{2},-\frac{1}{2} t+4\right] .
\end{gathered}
$$

It can be illustrated as in Figure 3.2.

As one can see,

$$
\begin{array}{cc}
W(T) \subset W(0), & W(T) \neq W(0), \\
W^{-}(T) \subset W^{-}(0), & W^{-}(T) \neq W^{-}(0),
\end{array}
$$

and $\{T\} \times W^{-}(T)$ is a strong deformation retract of $W^{-}$. Moreover, the sets $W(0)$, $W^{-}(0)$ are compact ANRs and $\Lambda\left(W, W^{-}\right)=-1 \neq 0$. Hence, there exists a viable 
6-periodic trajectory in $W$. Notice that the problem cannot be reduced to the one covered by the results from [14].

Corollary 3.15. Suppose that $\left(W, W^{-}\right)$is of the form $\left([0, T] \times K,[0, T] \times K^{-}\right)$for some compact ANRs $K$ and $K^{-}$. If $\chi(K) \neq \chi\left(K^{-}\right)$, then there is a viable T-periodic trajectory in W. Moreover, assuming (3.6), then

$$
\text { ind }\left(\varphi_{(0, T)}, P_{W}\right)=\chi(K)-\chi\left(K^{-}\right) \text {. }
$$

Sometimes, when trajectories start from more regular set than ANR, we can look for viable periodic trajectories studying only the map $H_{1}^{-}$as in the following.

Corollary 3.16. Let assumptions (3.3), (3.10), (3.12), and (3.19) be satisfied. If $W(0)$ is an absolute retract, then $\lambda\left(H_{1}^{-}\right) \neq 1$ implies that there exists a viable $T$-periodic trajectory in W. Moreover, if condition (3.6) holds, then

$$
\text { ind }\left(\varphi_{(0, T)}, P_{W}\right)=1-\lambda\left(H_{1}^{-}\right) \text {. }
$$

Corollary 3.17. Let assumptions (3.3), (3.10), (3.12), and (3.19) be satisfied. If $W(0)$ is an absolute retract and $W^{-}(T)$ consists of two disjoint absolute retracts $A_{1}$ and $A_{2}$, then there exists a viable $T$-periodic trajectory in $W$.

Proof. It is sufficient to notice that $\lambda\left(H_{1}^{-}\right)$equals 0 or 2 . Corollary 3.16 finishes the proof.

Below, we give an example that for arbitrary $A_{i}$ a viable $T$-periodic trajectory may not exist.

Example 3.18. Let

$$
\begin{aligned}
K:=\{ & (r \cos \psi, r \sin \psi, z) \in \mathbb{R}^{3} \mid \psi \in[0,2 \pi], r \in[0,3], \\
& -r+1 \leq z \leq 2 \text { for } r \in[0,1],-2 \leq z \leq 2 \text { for } r \in[1,3]\} .
\end{aligned}
$$

It means that we rotate around the $z$-axis the set $A$ in Figure 3.3.

Define a field $g_{1}(\psi, r, z):=(1, r(2-r), z)$ which induces the Lipschitz map $g$ on $\mathbb{R}^{3}$ (after changing coordinates).

The exit set for $K$ is the set $A_{s}:=\{(x, z) \in A|| z \mid=2\}$ rotated around the $z$-axis, that is, $K_{s}=\{(x, y, z) \in K|| z \mid=2\}$.

Now, consider the tube $W:=[0, T] \times K$, where $0<T<2 \pi$, and a field $f(t, u):=(1$, $g(u))$ for every $u \in \mathbb{R}^{3}$. Then, $W^{-}=[0, T] \times K_{s}$ and obviously $\{T\} \times W^{-}(T)$ is a strong deformation retract of $W^{-}$. Notice that, from Corollary 3.15, it follows that

$$
\Lambda\left(W, W^{-}\right)=\chi(K)-\chi\left(K^{-}\right)=1-1=0 .
$$




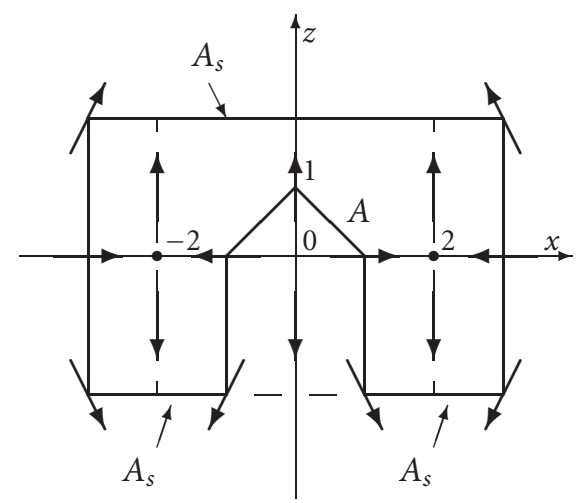

Figure 3.3

Notice that in $W$ there is only one trajectory with possible periodicity $\varphi_{(0, \psi)}(2 \cos \psi$, $2 \sin \psi, 0)$. Since $T<2 \pi$, it is not $T$-periodic.

For more than two absolute retracts $A_{i}$ in Corollary 3.17, it may also occur that $\Lambda(W$, $\left.W^{-}\right)=0$ (some of them change places). It seems that one can find examples that, moreover, there is no viable periodic trajectory. However, we can easily prove the following result in a low dimension.

Corollary 3.19. Let assumptions (3.3), (3.10), (3.12), and (3.19) be satisfied. If $n \leq 2$ (dimension of a space), $W(0)$ is an absolute retract and $W^{-}(T)$ consists of disjoint absolute retracts $A_{1}, \ldots, A_{k}, k \geq 2$, then there exists a viable T-periodic trajectory in $W$.

Proof. It is easy to see that, since the topological dimension of $\partial W$ is not greater than 2 , $\lambda\left(H_{1}^{-}\right)=0$ or $\lambda\left(H_{1}^{-}\right)=k \neq 1$. Therefore, $\Lambda\left(W, W^{-}\right) \neq 0$, which ends the proof.

We end this section giving the result on existence of viable $T$-periodic trajectories without assumption (3.10).

Proposition 3.20. Let $W \subset[0, T] \times \mathbb{R}^{n}$ be a right-continuous compact tube. If $W(0) \subset$ $W(T), W(T)$ is an absolute retract and $W^{-}=\operatorname{cl}(\partial W \backslash(\{T\} \times W(T)))$, then there is a viable $T$-periodic trajectory in $W$.

Proof. Consider the map $\tilde{f}: \mathbb{R} \times \mathbb{R}^{n} \rightarrow \mathbb{R}^{n}, \tilde{f}(t, x):=-f(t, x)$. Then, the exit set $\widetilde{W}^{-}$for $(-1, \tilde{f}(\cdot))$ is empty and each trajectory starting from $W(T)$ is viable with $\tilde{\varphi}_{(0, T)}(x):=$ $\varphi_{(T,-T)}(x) \in W(0) \subset W(T)$.

By the Schauder fixed point theorem, there exists a point $x_{0} \in W(T)$, and in fact in $W(0)$, such that $\tilde{\varphi}_{(0, T)}\left(x_{0}\right)=x_{0}$. Now, the trajectory $\varphi_{(0, \cdot)}\left(x_{0}\right)$ is the one we look for. The proof is complete.

One can easily find examples that there may be no viable $T$-periodic solutions if $W(0) \subset W(T), W(0) \neq W(T)$, and $W^{-}$is not a whole boundary. 


\section{Differential inclusions}

The problem of finding $T$-periodic trajectories for differential inclusions is much more complicated. While there are some techniques (see, e.g., [13]) for solving this problem when on the boundary of the tube the tangential condition $(\{1\} \times F(t, x)) \cap T_{W}(t, x) \neq \varnothing$ is satisfied, the situation becomes dramatically worse if this condition does not hold. For instance, we cannot prove that the solution map assigning to a point $(t, x) \in W$ the set of viable solutions starting from it has sufficiently regular values.

The idea is to approximate our multivalued map by sufficiently regular single-valued maps having $T$-periodic trajectories. However, we should follow in such a way that the topological properties of all exit sets for these approximating maps would be under control, which is very difficult, or even impossible, in general.

Nevertheless, as we can see, in some situations we can overcome these difficulties.

The first result we present is self-evident and we include it only for making our discussion more complete. We will use for a tube $W \subset[0, T] \times \mathbb{R}^{n}$ the following set:

$$
\begin{aligned}
W^{e} & :=\left(W_{e} \backslash(\{T\} \times W(T))\right) \\
& \cup\left\{(T, x) \in\{T\} \times W(T) \mid \exists\left(t_{m}, x_{m}\right) \in W_{e} \backslash(\{T\} \times W(T)):\left(t_{m}, x_{m}\right) \longrightarrow(T, x)\right\},
\end{aligned}
$$

where $W_{e}$ was defined in Section 2.

Proposition 4.1. Assume that a Marchaud map $F$ and a compact right-continuous tube $W \subset[0, T] \times \mathbb{R}^{n}$ satisfy assumptions (3.10), (3.12), (3.19), and $F$ possesses measurable Lipschitz selections, and $W^{e}=W^{-}$. Then, $\Lambda\left(W, W^{-}\right) \neq 0$ implies that there exists a viable T-periodic trajectory for $F$ in $W$. (If $F(t, \cdot)$ is Lipschitz, that is, $d_{H}(F(t, x), F(t, y)) \leq$ $L|x-y|$, where $d_{H}$ stands for the Hausdorff metric, then obviously $f(t, x):=\sigma(F(t, x))$, where $\sigma(F(t, x))$ denotes the Steiner point of $F(t, x)$, is a measurable Lipschitz selection of $F$.)

It is obvious that each measurable Lipschitz selection of $F$ satisfies all assumptions of Theorem 3.11 and a viable $T$-periodic trajectory for $f$ is a desired solution.

The assumption $W^{e}=W^{-}$for multivalued maps is strong while it is evidently verified in problems with the uniqueness property. In what follows, we avoid this condition. However, some regularity assumptions on the tube are necessary.

Definition 4.2. A right-continuous compact tube $W \subset[0, T] \times \mathbb{R}^{n}$ is an $(n+1) C^{1,1}$ tube if there exists an extended right-continuous tube $\widehat{W} \subset(a, b) \times \mathbb{R}^{n}, a \leq 0 \leq T \leq b \leq+\infty$ (i.e., $\widehat{W}(t)=W(t)$ for every $t \in[0, T])$, which is a $C^{1,1}(n+1)$-manifold with a boundary $\partial \widehat{W}$.

$\left(W, W^{-}\right)$is an $(n+1) C^{1,1}$ pair if $W$ is an $(n+1) C^{1,1}$ tube and the exit set $\widehat{W}_{s}$ for $\{1\} \times F(\cdot)$, if it is nonempty, is a $C^{1,1} n$ submanifold of $\partial \widehat{W}$ with a boundary $\partial_{\partial \widehat{W}} \widehat{W}_{s}$, and $\widehat{W}_{s} \cap W=W^{-}$.

We will assume that the map $(t, x) \multimap F(t, x)$ is continuous. Weaker assumptions are left for further research. Our present aim is to show a possible method of studying the existence of viable $T$-periodic trajectories which consists in a controlled approximation of a right-hand side $F$ by sufficiently regular single-valued maps. 


\section{Periodic solutions in tubes}

Our technique is based on the following crucial result.

Lemma 4.3. Let $K, X$, and $F: \mathbb{R}^{n} \rightarrow \mathbb{R}^{n}$ satisfy the following:

(I) $K$ is a $C^{1,1} n$-manifold with a boundary, and $X \subset K$ is a compact subset;

(II) $F$ is a continuous Marchaud map;

(III) there is a compact neighborhood $P$ of $X$ in $K$ such that $K_{s}(F) \cap P$ is closed;

(IV) if $K_{s}(F)$ is nonempty, it is a $C^{1,1}(n-1)$-submanifold of $\partial K$ with a boundary $\partial_{\partial K} K_{s}(F)$.

Then, for every $\varepsilon>0$, there exists $f: \mathbb{R}^{n} \rightarrow \mathbb{R}^{n}$ such that

(a) $f$ is an $\varepsilon$-approximation of $F(f: X \rightarrow Y$ is an $\varepsilon$-approximation of a compact valued map $F: X \multimap Y$, if, for every $x \in X, f(x) \in B(z, \varepsilon)$ for some $z \in F(B(x, \varepsilon)))$;

(b) $K_{s}(f) \cap X=K_{s}(F) \cap X$;

(c) $f$ is Lipschitz on some open neighborhood of $X$ in $\mathbb{R}^{n}$.

Proof. Let $S \subset K$ be a compact neighborhood of $X$ such that $S \subset \operatorname{Int}_{K} P$. We proceed in two steps.

Step 1. We construct an open set $U$ in $\mathbb{R}^{n}$ such that $S \backslash K_{s}(F) \subset U$ and $\left(K_{s}(F) \cap S\right) \cap U=$ $\varnothing$, and a map $g: U \rightarrow \mathbb{R}^{n}$ such that

(A) $\left\langle g(x), v_{x}\right\rangle<0$ for every $x \in\left(\partial K \backslash K_{s}(F)\right) \cap S$;

(B) $g$ is $C^{\infty}$ in $U$;

(C) $g(x) \in F(x)+\min \left\{\varepsilon, d_{K_{s}(F)}(x)\right\} B_{1}$ for every $x \in S \backslash K_{s}(F)$.

To do this, at first we find for every $x \in S \backslash K_{s}(F)$ a vector $v_{x} \in \mathbb{R}^{n}$ and an open neighborhood $U_{x}$ of $x$ in $\mathbb{R}^{n}$ in the following way.

Case 1. Let $x \in\left(\partial K \backslash K_{s}(F)\right) \cap S$. Then, there exists $w_{x} \in F(x) \cap T_{K}(x)$. Denote $\eta_{x}:=$ $d_{K_{s}(F)}(x)>0$ and $\varepsilon_{x}:=(1 / 4) \min \left\{\varepsilon, \eta_{x}\right\}$.

Take an open neighborhood $U_{x}$ of $x$ such that $d_{K_{s}(F)}(y) \geq \eta_{x} / 2$ and $F(x) \subset F(y)+\varepsilon_{x} B_{1}$, for every $y \in U_{x}$.

Now, take $v_{x}:=w_{x}-t_{x} v_{x}\left(t_{x}>0\right.$ is sufficiently small) such that $v_{x} \in F(x)+\varepsilon_{x} B_{1}$.

Then, for $y \in U_{x} \cap K$,

$$
\begin{gathered}
v_{x} \in F(y)+2 \varepsilon_{x} B_{1} \subset F(y)+\frac{1}{2} \eta_{x} B_{1} \subset F(y)+d_{K_{s}(F)}(y) B_{1}, \\
v_{x} \in F(y)+\frac{\varepsilon}{2} B_{1} .
\end{gathered}
$$

Moreover, we can take $U_{x}$ so small that $\left\langle v_{x}, v_{y}\right\rangle<0$ for every $y \in U_{x} \cap \partial K$, since $\left\langle v_{x}\right.$, $\left.v_{x}\right\rangle=\left\langle w_{x}, v_{x}\right\rangle-t_{x}<0$.

Case 2. Let $x \in S \backslash \partial K$. Similarly as above, we can find $U_{x}$ with $U_{x} \cap \partial K=\varnothing$ and $v_{x} \in$ $F(x)$ satisfying (4.2) and (4.3) for any $y \in U_{x}$.

Choose a countable, locally finite covering $\left\{U_{x_{i}} \mid x_{i} \in S \backslash K_{s}(F)\right\}$ of $S \backslash K_{s}(F)$ and consider the smooth $\left(C^{\infty}\right)$ partition of unity $\left\{\lambda_{i}: U_{x_{i}} \rightarrow[0,1]\right\}$ subordinated to it.

Define $U:=\bigcup_{i=1}^{\infty} U_{x_{i}}$ and notice that $S \backslash K_{s}(F) \subset U$ and $U \cap K_{s}(F)=\varnothing$.

For every $x \in U$, denote $I(x):=\left\{i \in \mathbb{N} \mid \lambda_{i}(x) \neq 0\right\}$ and define

$$
g(x):=\sum_{i \in I(x)} \lambda_{i}(x) v_{x_{i}}
$$


Since each $\lambda_{i}$ is $C^{\infty}$, (B) holds.

To verify (A), take $x \in\left(\partial K \backslash K_{s}(F)\right) \cap S$ and notice that, by the definition of $v_{x_{i}}$ and $U_{x_{i}}$, for each $i \in I(x)$, one has $x_{i} \in\left(\partial K \backslash K_{s}(F)\right) \cap S$ and

$$
\left\langle g(x), v_{x}\right\rangle=\sum_{i \in I(x)} \lambda_{i}(x)\left\langle v_{x_{i}}, v_{x}\right\rangle<0 .
$$

Now, for $x \in S \backslash K_{s}(F)$ and for $i \in I(x)$, by (4.2), one has $v_{x_{i}} \in F(x)+d_{K_{s}(F)}(x) B_{1}$ and this set is convex. Hence,

$$
g(x)=\sum_{i \in I(x)} \lambda_{i}(x) v_{x_{i}} \in F(x)+d_{K_{s}(F)}(x) B_{1} .
$$

Similarly, $g(x) \in F(x)+(\varepsilon / 2) B_{1}$, so $(C)$ is also satisfied.

Step 2. In order to construct the map $f$ we first define, for $x \in K_{s}(F) \cap S$, a map $v_{x}(\cdot)$ and an open neighborhood $U_{x}$ of $x$ in the following way.

Case 1. Let $x \in \operatorname{Int}_{\partial K} K_{s}(F) \cap S$. We know that $\min _{v \in F(x)}\left\langle v, v_{x}\right\rangle \geq 0$.

Fix $w_{x} \in F(x)$, so $\left\langle w_{x}, v_{x}\right\rangle \geq 0$. Take an open neighborhood $U_{x}$ of $x$ in $\mathbb{R}^{n}$ such that $U_{x} \cap \partial_{\partial K} K_{s}(F)=\varnothing, U_{x} \cap \partial K \subset K_{s}(F)$ and $F(x) \subset F(y)+(\varepsilon / 2) B_{1}$, for every $y \in U_{x}$.

Define $v_{x}:=w_{x}+t_{x} v_{x}$, where $t_{x}>0$ is so small that $\left\langle v_{x}, v_{y}\right\rangle>0$ for $y \in U_{x} \cap K_{s}(F)$ and $v_{x} \in F(x)+(\varepsilon / 2) B_{1}$. Then, condition (4.3) holds.

Case 2. Suppose that $x \in \partial_{\partial K} K_{s}(F) \cap S$. Then, since $x \in K_{s}(F) \cap P$ which is closed, we know by Proposition 2.2 that there is $v \in F(x) \cap T_{K}(x)$ such that $v \in T_{K_{s}(F)}(x)$. There is an open neighborhood $V_{x}$ of $x$ in $\mathbb{R}^{n}$ and a $C^{1,1}$ diffeomorphism $\phi: V_{x} \rightarrow \phi\left(V_{x}\right) \subset \mathbb{R}^{n}$ such that $V_{x} \cap K_{s}(F) \subset P, \phi(x)=0$,

$$
\begin{gathered}
\phi\left(V_{x} \cap K\right)=\left\{\left(y_{1}, \ldots, y_{n}\right) \in \mathbb{R}^{n} \mid y_{1} \leq 0\right\}=: X, \\
\phi\left(V_{x} \cap K_{s}(F)\right)=\left\{\left(y_{1}, \ldots, y_{n}\right) \in X \mid y_{1}=0, y_{2} \geq 0\right\}=: X_{s} .
\end{gathered}
$$

As a consequence, for $z \in V_{x} \cap K$,

$$
\begin{gathered}
\operatorname{Int} \phi^{\prime}(z)\left(T_{K}(z)\right)=\left\{\left(y_{1}, \ldots, y_{n}\right) \in X \mid y_{1}<0\right\}, \\
\operatorname{Int} \phi^{\prime}(z)\left(T_{\mathbb{R}^{n} \backslash K}(z)\right)=\left\{\left(y_{1}, \ldots, y_{n}\right) \in \mathbb{R}^{n} \mid y_{1}>0\right\},
\end{gathered}
$$

and, for $z \in V_{x} \cap \partial_{\partial K} K_{s}(F)$,

$$
\operatorname{Int}_{\partial X} \phi^{\prime}(z)\left(T_{K_{s}(F)}(z)\right)=\left\{\left(y_{1}, \ldots, y_{n}\right) \in X \mid y_{1}=0, y_{2}>0\right\}
$$

Define on $\phi\left(V_{x}\right)$ the map $G(y):=\phi^{\prime}\left(\phi^{-1}(y)\right) F\left(\phi^{-1}(y)\right)$.

Let $\zeta(y):=\left(y_{2}, 1,0, \ldots, 0\right)$ and take $w:=\phi^{\prime}(x) v$. Then, $\left\langle w, e_{1}\right\rangle=0$ and $\left\langle w, e_{2}\right\rangle \geq 0$, where $e_{1}, e_{2}$ are unit vectors on two first axes. Let $u_{\theta}(y):=w+\theta \zeta(y)$, where $\theta>0$. Then,

$$
\begin{array}{cc}
\left\langle u_{\theta}(y), e_{1}\right\rangle<0 & \text { for } y \in \partial X \backslash X_{s}, \\
\left\langle u_{\theta}(y), e_{1}\right\rangle>0 & \text { for } y \in \operatorname{Int}_{\partial X} X_{s}, \\
\left\langle u_{\theta}(y), e_{2}\right\rangle>0 & \text { for } y \in \partial_{\partial X} X_{s} .
\end{array}
$$


Define, for $z \in V_{x}, v_{\theta}(z):=\left(\phi^{-1}\right)^{\prime}(\phi(z)) u_{\theta}(\phi(z))$. Using (4.10) and the fact that tangent cones correspond to tangent cones under the diffeomorphism $\phi$ (see (4.8), (4.9)), we obtain

$$
\begin{array}{cc}
\left\langle v_{\theta}(z), v_{z}\right\rangle<0 & \text { for } z \in\left(\partial K \backslash K_{s}(F)\right) \cap V_{x}, \\
\left\langle v_{\theta}(z), v_{z}\right\rangle>0 & \text { for } z \in\left(\operatorname{Int}_{\partial K} K_{s}(F)\right) \cap V_{x}, \\
\left\langle v_{\theta}(z), n_{z}\right\rangle>0 & \text { for } z \in\left(\partial_{\partial K} K_{s}(F)\right) \cap V_{x},
\end{array}
$$

where $n_{z}$ is an inward normal vector to $K_{s}(F)$ in $T_{\partial K}(z)$.

Similarly as in Case 1, we can find an open neighborhood $U_{x} \subset V_{x}$ of $x$ and a small $\theta_{x}>0$ such that, for $v_{x}(z):=v_{\theta_{x}}(z)$, we additionally have

$$
v_{x}(z) \in F(z)+\varepsilon B_{1} \quad \text { for every } z \in U_{x} \cap K \text {. }
$$

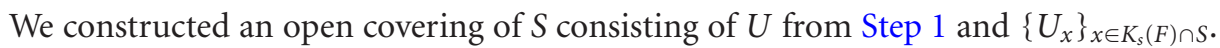
Since $K_{s}(F) \cap S$ is compact, we can choose a finite subcovering $\left\{U_{i}\right\}_{i=0}^{k}$, where $U_{0}:=U$, and consider a smooth partition of unity $\left\{\beta_{i}\right\}$ subordinated to it.

Define

$$
f_{0}(x):=\beta_{0}(x) g(x)+\sum_{i=1}^{k} \beta_{i}(x) v_{x_{i}}(x)
$$

Take any continuous selection of $F$ and its locally Lipschitz $\mathcal{E}$-approximation $f_{1}$. Take also open neighborhoods $\Omega, \Omega_{1}$ of $S$ in $\mathbb{R}^{n}$ such that $\bar{\Omega} \subset \Omega_{1} \subset \overline{\Omega_{1}} \subset \bigcup_{i=0}^{k} U_{i}$. Let $u: \mathbb{R}^{n} \rightarrow$ $[0,1]$ be a locally Lipschitz function such that $u \equiv 0$ on $\mathbb{R}^{n} \backslash \Omega_{1}$ and $u \equiv 1$ on $\Omega$. We are in a position to define $f: \mathbb{R}^{n} \rightarrow \mathbb{R}^{n}$,

$$
f(x):=u(x) f_{0}(x)+(1-u(x)) f_{1}(x) .
$$

We verify conditions (a)-(c).

The considerations above imply that $f_{i}(x) \in F(x)+\varepsilon B_{1}$. Since values of $F$ are convex, we have $f(x) \in F(x)+\varepsilon B_{1}$ and condition (a) is verified.

We check (b). Let $x \in\left(\partial K \backslash K_{s}(F)\right) \cap X$ and $I(x):=\left\{i \in\{1, \ldots, k\} \mid \beta_{i}(x) \neq 0\right\}$. From (4.11) and (A) it follows that, for $i \in I(x),\left\langle v_{x_{i}}(x), v_{x}\right\rangle<0$ and, consequently, $\left\langle g(x), v_{x}\right\rangle<0$. Therefore, $\left\langle f(x), v_{x}\right\rangle<0$, which implies that $K_{s}(f) \cap X \subset K_{s}(F) \cap X$.

Notice that, for $x \in \operatorname{Int}_{\partial K} K_{s}(F) \cap X, \beta_{0}(x)=0$ and $\left\langle v_{x_{i}}, v_{x}\right\rangle>0$ (see Step 2, Case 1). Thus, $\left\langle f(x), v_{x}\right\rangle>0$. If $x \in \partial_{\partial K} K_{s}(F) \cap X$, then $x_{i} \in \partial_{\partial K} K_{s}(F)$ for every $i \in I(x)$. Moreover, $\beta_{0}(x)=0,\left\langle v_{x_{i}}(x), v_{x}\right\rangle=0$ and $\left\langle v_{x_{i}}, n_{x}\right\rangle>0$, which implies that $\left\langle f(x), n_{x}\right\rangle>0$ while $\left\langle f(x), v_{x}\right\rangle=0$. From Proposition 2.2 it follows that a trajectory for $f$ starting from $x$ leaves the set $K$ immediately. Therefore, $K_{s}(f)=K_{s}(F)$.

Finally, it is seen that $f$ is locally Lipschitz according to regularity of $\beta_{i}$ and the diffeomorphism $\phi$; the proof is complete. 
We can adopt Lemma 4.3 to a nonautonomous case and obtain the following lemma.

Lemma 4.4. Let $F: \mathbb{R} \times \mathbb{R}^{n} \multimap \mathbb{R}^{n}$ be a continuous Marchaud map with a growth $|F(t, x)| \leq$ $c(1+|x|)$, and let $\left(W, W^{-}\right)$be an $(n+1) C^{1,1}$ pair. Then, for every $\varepsilon>0$, there exists a map $f: \mathbb{R} \times \mathbb{R}^{n} \rightarrow \mathbb{R}^{n}$ such that

(a) the exit sets for $f$ and $F$ are the same;

(b) $f$ is an $\varepsilon$-approximation of $F$ in some neighborhood of $W$ where it is Lipschitz.

Proof. Let $\widehat{W}$ be an extended $C^{1,1}$ tube of $W$ and $\widehat{W}_{s}$ its exit set. Consider the continuous $\operatorname{map} G: \mathbb{R} \times \mathbb{R}^{n} \multimap \mathbb{R} \times \mathbb{R}^{n}, G(t, x):=\{1\} \times F(t, x)$.

Take an arbitrary $0<\varepsilon<1$. Let

$$
M:=\sup \left\{|y|+1 \mid y \in F(t, x),(t, x) \in W+B_{1}\right\}
$$

and $\delta:=\min \{\varepsilon /(1+2 M), 1 / 2\}$.

From Lemma 4.3 it follows that there is a map $g: \mathbb{R} \times \mathbb{R}^{n} \rightarrow \mathbb{R} \times \mathbb{R}^{n}$ which is a $\delta$ approximation of $G$, Lipschitz on some open neighborhood $\Omega \subset W+B_{1}$ of $W$, and such that $W^{-}=\widehat{W}_{s} \cap W$ is the exit set for $g$ on $W$. Denote $g(t, x)=\left(g_{1}(t, x), g_{2}(t, x)\right)$, where $g_{1}(t, x) \in \mathbb{R}$ and $g_{2}(t, x) \in \mathbb{R}^{n}$.

Define $f: \mathbb{R} \times \mathbb{R}^{n} \rightarrow \mathbb{R}^{n}$,

$$
f(t, x):=\frac{1}{g_{1}(t, x)} g_{2}(t, x)
$$

It is easy to see that the exit set for $f$ equals $W^{-}$, since $(1, f(t, x))=\left(1 / g_{1}(t, x)\right) g(t, x)$. Moreover, $f$ is a well-defined $\varepsilon$-approximation of $F$, Lipschitz on $\Omega$. Indeed, the Lipschitzeanity is obvious, since $\left|g_{1}(t, x)-1\right|<1 / 2$. Let $(t, x) \in \Omega$. Since $g$ is a $\delta$-approximation of $G$, there is $y \in F(s, z)$ for some $|s-t|<\delta$ and $|z-x|<\delta$ such that

$$
\left|g_{1}(t, x)-1\right|<\delta, \quad\left|g_{2}(t, x)-y\right|<\delta .
$$

It implies that

$$
\begin{aligned}
|f(t, x)-y| & =\left|\frac{1}{g_{1}(t, x)} g_{2}(t, x)-y\right| \\
& \leq\left|\frac{1}{g_{1}(t, x)} g_{2}(t, x)-g_{2}(t, x)\right|+\left|g_{2}(t, x)-y\right| \\
& <\frac{\left|1-g_{1}(t, x)\right|}{g_{1}(t, x)}\left|g_{2}(t, x)\right|+\delta<2 M \delta+\delta \leq \varepsilon,
\end{aligned}
$$

which means that $f$ is an $\mathcal{\varepsilon}$-approximation of $F$. The proof is complete.

Using the above lemma, we can prove the main result of this section.

Theorem 4.5. Let $F: \mathbb{R} \times \mathbb{R}^{n} \multimap \mathbb{R}^{n}$ be a continuous Marchaud map with a growth $\mid F(t$, $x) \mid \leq c(1+|x|)$, and let $\left(W, W^{-}\right)$be an $(n+1) C^{1,1}$ pair. Assume that $W(T) \subset W(0)$, $W^{-}(T) \subset W^{-}(0)$ and $\{T\} \times W^{-}(T)$ is a strong deformation retract of $W^{-}$. If $\Lambda\left(W, W^{-}\right) \neq$ 0 , then there exists a viable $T$-periodic trajectory for $F$ in $W$. 
Proof. Let $\left\{\varepsilon_{m}\right\}$ be a sequence of positive numbers converging to 0 . For each $m \geq 1$, following Lemma 4.4, we find a Lipschitz $\varepsilon_{m}$-approximation $f_{m}$ of $F$ generating the same exit set $W^{-}$.

Using Theorem 3.11, we can find $x_{m} \in W(0)$ such that the trajectory $\bar{x}_{m}(\cdot)$ for $f_{m}$ starting from $x_{m}$ is viable and $T$-periodic. Since $W(0)$ is compact, without any loss of generality we can assume that $x_{m} \rightarrow x \in W(0)$.

Now, we use the fact that $F$ has at most a linear growth and $W$ is compact, and, from the condition

$$
\dot{\bar{x}}_{m}(t)=f_{m}\left(t, \bar{x}_{m}(t)\right) \in F\left(B\left(\left(t, \bar{x}_{m}(t)\right), \frac{1}{m}\right)\right)+\frac{1}{m} B_{1},
$$

we deduce that there is a constant $C>0$ such that $\left|\bar{x}_{m}(t)\right| \leq C$ for every $t \in[0, T]$ and $\left|\dot{\bar{x}}_{m}(t)\right| \leq C$ for almost every $t \in[0, T]$. By the convergence theorem (see [1, Theorem $0.3 .4]$ ), we can assume that $\bar{x}_{m} \rightarrow x$ uniformly, and $\dot{\bar{x}}_{m}$ converges weakly in $L^{1}$ to $\dot{x}$ for some absolutely continuous map $x$. This implies that $x$ is viable in $W$ and $x(0)=x(T)$. It is sufficient to prove that $\dot{x}(t) \in F(t, x(t))$ a.e. in $[0, T]$.

Notice that $\dot{x}$ belongs to the weak closure of the set $\operatorname{conv}\left\{\dot{\bar{x}}_{m} \mid m \geq k\right\}$. By the Mazur lemma, it also belongs to the strong closure of this set. Hence, there is a sequence $z_{k} \in$ $\operatorname{conv}\left\{\dot{\bar{x}}_{m} \mid m \geq k\right\}$ such that $\left\|z_{k}-\dot{x}\right\|_{L^{1}} \leq 1 / k$. This implies that there exists a subsequence $z_{k_{l}} \rightarrow \dot{x}$ a.e. in $[0, T]$.

Take an arbitrary $\varepsilon>0$, and let $s \in[0, T]$ be such that $\lim _{l \rightarrow \infty} z_{k_{l}}(s)=\dot{x}(s)$. There exists $\delta>0, \delta<\varepsilon$, such that $F(s, y) \subset F(s, x(s))+\varepsilon B_{1}$ if $|y-x(s)|<\delta$. On the other hand, there is $N \geq 1$ such that $1 / N<\delta / 2$ and $\left|\bar{x}_{m}(s)-x(s)\right|<\delta / 2$ for every $m \geq N$ and $s \in[0, T]$. Therefore,

$$
\dot{\bar{x}}_{m}(s) \in F(B((s, x(s)), \delta))+\frac{\delta}{2} B_{1} \subset F(s, x(s))+2 \varepsilon B_{1} .
$$

Hence, $z_{k_{l}}(s) \in F(s, x(s))+2 \varepsilon B_{1}$ for every $l \geq 1$, which implies that $\dot{x}(s) \in F(s, x(s))+$ $2 \varepsilon \overline{B_{1}}$. Since $\varepsilon$ was arbitrary, one has $\dot{x}(s) \in F(s, x(s))$ for almost every $s \in[0, T]$. This finishes the proof.

As a consequence, we can state the following corollary.

Corollary 4.6. Consider the control problem with constraints

$$
\dot{x}=f(t, x, u), \quad u \in U \subset \mathbb{R}^{m}, \quad(t, x) \in W \subset[0, T] \times \mathbb{R}^{n}, \quad x(0)=x(T),
$$

and assume that the map $F(t, x):=f(\{(t, x)\} \times U)$ is a continuous Marchaud map. If $\left(W, W^{-}\right)$forms an $(n+1) C^{1,1}$ pair with $W(0), W^{-}(0)$ being $A N R s, W(T) \subset W(0)$, $W^{-}(T) \subset W^{-}(0)$, and such that $\{T\} \times W^{-}(T)$ is a strong deformation retract of $W^{-}$, then $\Lambda\left(W, W^{-}\right) \neq 0$ implies that there is a control $u:[0, T] \rightarrow U$ inducing a solution $x(\cdot)$ to problem (4.21). 


\section{Acknowledgment}

The author would like to express his thanks to Professors Marc Quincampoix and Pierre Cardaliaguet for valuable discussions and comments, and fruitful collaboration during the author's stay in Brest.

\section{References}

[1] J.-P. Aubin and A. Cellina, Differential Inclusions: Set-Valued Maps and Viability Theory, Grundlehren der Mathematischen Wissenschaften, vol. 264, Springer-Verlag, Berlin, 1984.

[2] K. Borsuk, Theory of Retracts, Monografie Matematyczne, vol. 44, Państwowe Wydawnictwo Naukowe, Warsaw, 1967.

[3] R. F. Brown, The Lefschetz Fixed Point Theorem, Scott, Foresman and Co., Illinois, 1971.

[4] P. Cardaliaguet, Conditions suffisantes de non-vacuité du noyau de viabilité [Sufficient conditions for nonemptiness of the viability kernel], C. R. Acad. Sci. Paris Sér. I Math. 314 (1992), no. 11, 797-800.

[5] P. Cardaliaguet, G. Gabor, and M. Quincampoix, Equilibria and strict equilibria of multivalued maps on noninvariant sets, Ann. Polon. Math. 82 (2003), no. 1, 19-37.

[6] F. S. De Blasi and J. Myjak, On the solutions sets for differential inclusions, Bull. Polish Acad. Sci. Math. 33 (1985), no. 1-2, 17-23.

[7] G. Dylawerski and L. Górniewicz, A remark on the Krasnosiel'skiı̌s translation operator along trajectories of ordinary differential equations, Serdica 9 (1983), no. 1, 102-107.

[8] G. Gabor and R. Pietkun, Periodic solutions of differential inclusions with retards, Topol. Methods Nonlinear Anal. 16 (2000), no. 1, 103-123.

[9] L. Górniewicz, Topological Fixed Point Theory of Multivalued Mappings, Mathematics and Its Applications, vol. 495, Kluwer Academic Publishers, Dordrecht, 1999.

[10] M. A. Krasnosel'skiŭ, The Operator of Translation along the Trajectories of Differential Equations, Translations of Mathematical Monographs, vol. 19, American Mathematical Society, Rhode Island, 1968, translated from Russian by Scripta Technica.

[11] W. Kryszewski, Constrained differential inclusions, Set Valued Mappings with Applications in Nonlinear Analysis, Ser. Math. Anal. Appl., vol. 4, Taylor \& Francis, London, 2002, pp. 227 249.

[12] S. Plaskacz, Periodic solutions of differential inclusions on compact subsets of $\mathbf{R}^{n}$, J. Math. Anal. Appl. 148 (1990), no. 1, 202-212.

[13] On the solution sets for differential inclusions, Boll. Un. Mat. Ital. A (7) 6 (1992), no. 3, 387-394.

[14] R. Srzednicki, Periodic and bounded solutions in blocks for time-periodic nonautonomous ordinary differential equations, Nonlinear Anal. 22 (1994), no. 6, 707-737.

[15] R. Srzednicki and K. Wójcik, A geometric method for detecting chaotic dynamics, J. Differential Equations 135 (1997), no. 1, 66-82.

[16] T. Ważewski, Sur un principe topologique de l'examen de l'allure asymptotique des intégrales des équations différentielles ordinaires, Ann. Soc. Polon. Math. 20 (1947), 279-313.

[17] K. Wójcik, Periodic segments and Nielsen numbers, Conley Index Theory (Warsaw, 1997), Banach Center Publ., vol. 47, Polish Academy of Sciences, Warsaw, 1999, pp. 247-252.

[18] K. Wójcik and P. Zgliczyński, Isolating segments, fixed point index, and symbolic dynamics, J. Differential Equations 161 (2000), no. 2, 245-288.

Grzegorz Gabor: Faculty of Mathematics and Computer Science, Nicolaus Copernicus University, Chopina 12/18, 87-100 Torun, Poland

E-mail address: ggabor@mat.uni.torun.pl 


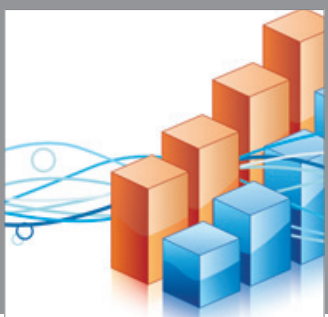

Advances in

Operations Research

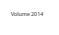

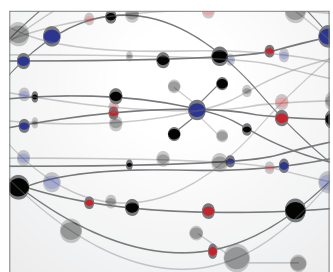

\section{The Scientific} World Journal
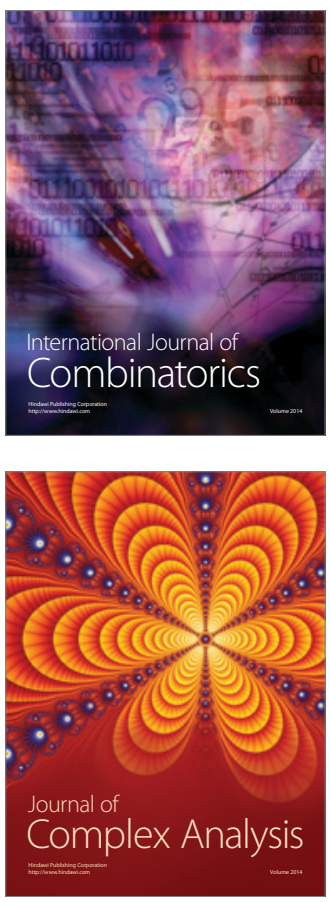

International Journal of

Mathematics and

Mathematical

Sciences
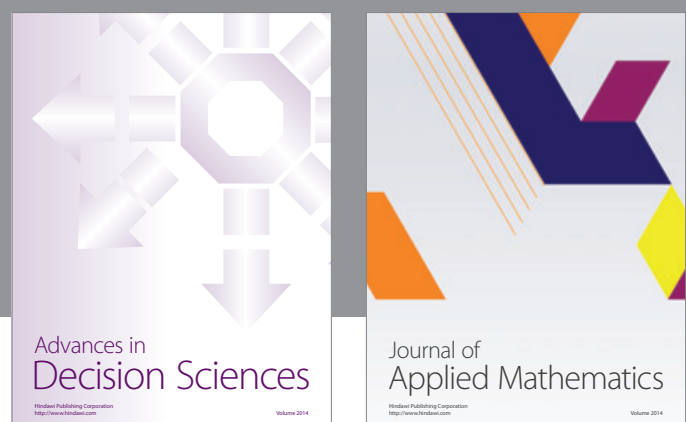

Journal of

Applied Mathematics
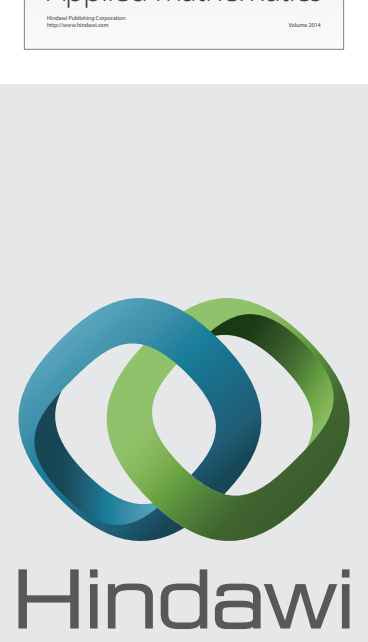

Submit your manuscripts at http://www.hindawi.com
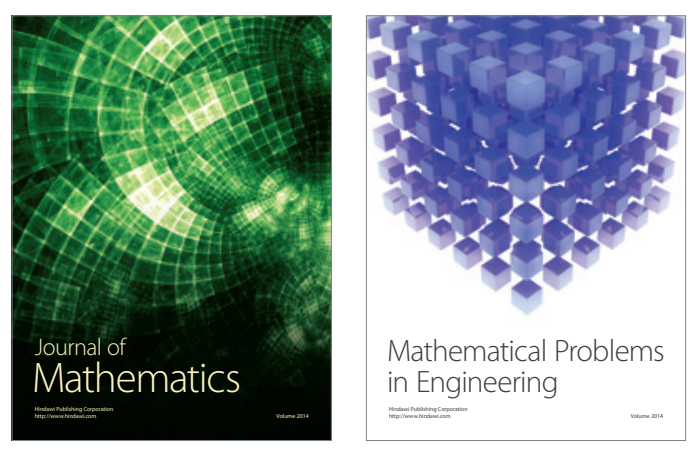

Mathematical Problems in Engineering
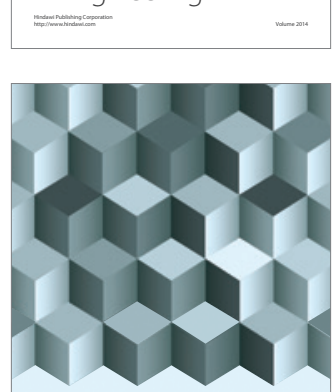

Journal of

Function Spaces
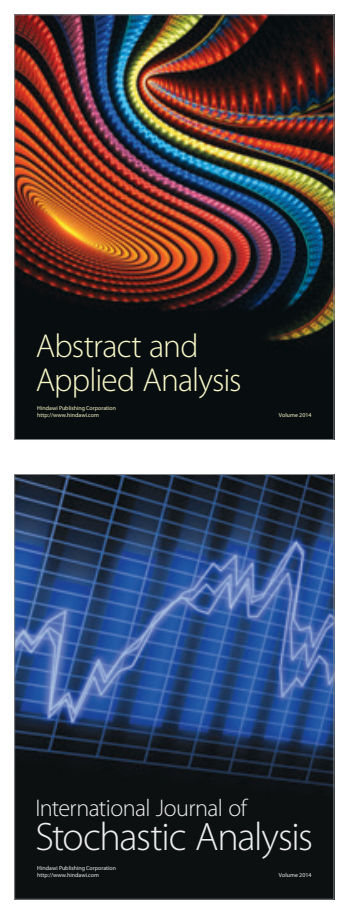

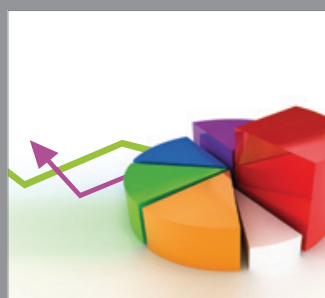

ournal of

Probability and Statistics

Promensencen
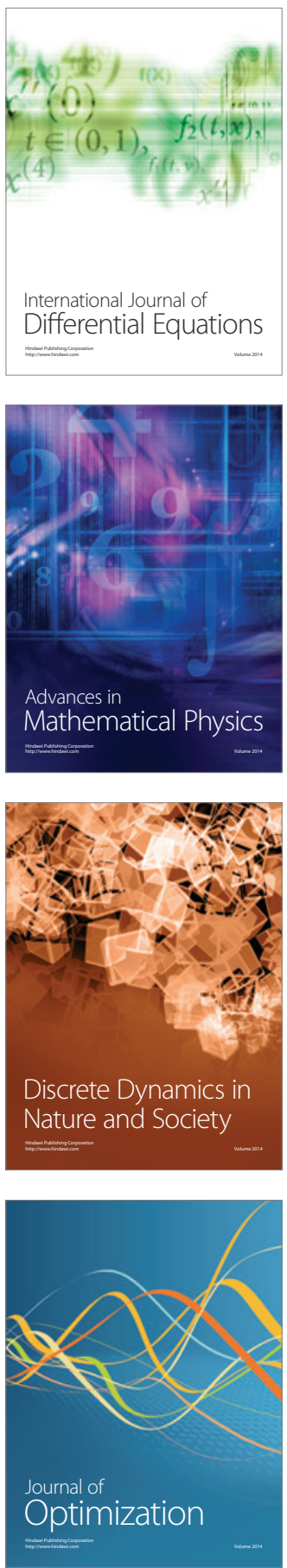